\title{
Recursos de información electrónicos en los Centros de Documentación Europea: el caso de España
}

\author{
Yolanda Martín González *
}

Artículo recibido:

19 de marzo de 2013.

Artículo aceptado:

7 de agosto de 2013.

\section{Resumen}

El propósito de este trabajo es esbozar el mapa de recursos electrónicos que ofrecen los Centros de Documentación Europea con el fin de proporcionar una guía de fuentes de información especializadas en la Unión Europea, que sirva de herramienta a aquellas personas interesadas en esta temática.

Para la realización del estudio se eligieron como muestra los 37 centros establecidos en instituciones españolas de enseñanza superior. A través del análisis de sus portales web, se perfiló el amplio conjunto de recursos informativos que aquellos ofrecen y se procedió posteriormente a organizarlos.

Tras la elaboración del trabajo y, a tenor de los resultados alcanzados, podemos concluir que los centros de

* Universidad de Salamanca, España. ymargon@usal.es 
documentación europea conforman una importante herramienta en la búsqueda y recuperación de información de la Unión Europea dado el amplio y variado número de recursos que permiten consultar. Sin embargo, se constata una fuerte tendencia a ofrecer fuentes oficiales de origen institucional y una notable escasez de recursos informativos elaborados por el propio centro.

Palabras clave: Centros de Documentación Europea; Unión Europea; Fuentes de información; Recursos electrónicos.

\section{Abstract}

Digital Information Resources in European Documentation Centres: A case study in Spain

Yolanda Martín-González

The purpose of this paper is to provide a guide map for persons interested of information sources offered by the European Documentation Centers and specialized electronic resources in the European Union. The study examined thirty-seven centers in Spanish institutions of higher learning. The web pages of these institutions were thoroughly inventoried and categorized, allowing the researcher to conclude that European documentation centers, by virtue of the diversity of information sources they gather in one place, constitute an important tool for users wishing to search and retrieve information on the European Union. The research also revealed a bias in information centers for institutional sources and a conspicuous scarcity of information resources produced in the centers themselves.

Keywords: European Documentation Centres; European Union; Information sources; Electronic resources.

\section{INTRODUCCIÓN}


de sus funciones. En el caso de la Unión Europea esta exigencia se enmarca en su política de información y comunicación desarrollada formalmente a partir de los años 90 del siglo XX. Con la aprobación del Tratado de la Unión Europea (1992) y del Tratado de Ámsterdam (1997), el principio de transparencia informativa y de derecho de acceso a la documentación institucional se convierten en pilares fundamentales de la actuación de la UE (Olarán y Pérez-Trompeta, 2000; Sánchez Domínguez, 2001).

No obstante, la preocupación por crear mecanismos difusores de las políticas y las actividades acometidas por las instituciones comunitarias se remonta a los años 60, fecha en que la Comisión Europea crea los Centros de Documentación Europea con el objeto de que sirvan de multiplicadores de información. A estos centros se unirán otros y juntos conformarán el sistema de información europeo con el que la UE pretende darle solución al problema del déficit informativo existente y que para muchos constituye la base del déficit democrático que amenaza a la Unión (Marcella, et al., 1997).

Su diferente tipología estará directamente relacionada con el público o con los sectores de la sociedad a los que se dirigen (Moreira, 2002): los Centros de Documentación Europea se encargarán de resolver las necesidades informativas en el medio universitario, los Euro Info Centres harán lo mismo en el ámbito empresarial, los Carrefours en el entorno rural y los Info Points se localizarán estratégicamente en las zonas urbanas.

En 2004 se procede a la reestructuración del sistema de información eurocomunitario y se define un modelo de enlace de información de segunda generación (Llorente Pinto, 2007): el servicio Europe Direct que aglutina a todos los centros de información hasta entonces existentes bajo una única coordinación, la Dirección General de Comunicación de la Comisión Europea.

Como ya hemos apuntado los Centros de Documentación Europea (CDE) constituyen una de las primeras redes de información creada por la Comisión Europea. Dichos centros ostentan una doble condición al actuar al mismo tiempo como unidades especializadas de información y como promotores de investigaciones y estudios académicos sobre la Europa Comunitaria. Además, los CDE se erigen en bibliotecas depositarias de los documentos y publicaciones oficiales de la Unión.

Al hallarse los CDE ubicados en el contexto universitario sus principales usuarios son docentes, investigadores, estudiantes y personal de administración y servicios, aunque también se encuentran al servicio del público en general. Precisamente, Terra (2010) ha realizado un interesante estudio sobre el perfil y las necesidades informativas propias de cada uno de estos grupos de usuarios en tanto que consumidores de documentación europea. 
Entre las distintas funciones asignadas a los centros de documentación se encuentra la de facilitar el acceso a las fuentes de información comunitarias y dirigir al ciudadano hacia aquellas que sean más adecuadas para resolver sus necesidades informativas. Además, estos centros poseen una importante dimensión formativa la cual se ve materializada con frecuencia en la impartición de cursos, organización de sesiones didácticas, jornadas especializadas, etcétera.

En la actualidad existen en el mundo 697 CDE de los cuales 403 se hallan ubicados en universidades y centros de investigación de los Estados miembros de la Unión. Del total de estos centros, 37 tienen su sede en España.

La implantación de CDE en nuestro país se desarrolla principalmente en los años 80, a partir de la entrada de España en la Comunidad Económica Europea. Algunos de los centros que reciben en primer lugar el estatuto de Centro de Documentación Europea son el de la Universidad de Deusto (1980) y el de la Universidad de Valladolid (1982).

Los CDE se benefician del envío gratuito y sistemático de la documentación institucional y oficial de la UE así como de las publicaciones editadas por la Oficina de Publicaciones Oficiales de las Comunidades Europeas. Con la creación en 1995 de Europa, el sitio web oficial de la UE, y la disposición de los documentos en formato digital, el papel de los CDE ha tenido que ser replanteado.

Por otra parte, la Comisión Europea no les proporciona a estos centros ningún tipo de directrices comunes para la creación de sus portales web, por lo que le corresponde a cada unidad de información determinar los recursos y servicios electrónicos que van a ofrecer a sus usuarios. En este sentido, tal y como afirma Terra (2009), las especificidades de cada país quedan reflejadas en las formas de organización de la información proporcionada digitalmente por los centros. A ello cabría añadir la influencia que a menudo esto posee sobre el tipo de recursos y/o información que ofrecen los centros, la facultad o el departamento universitario encargado de su gestión.

A pesar de que los centros de documentación europea están presentes en países de todo el mundo, existen muy pocos estudios sobre ellos, lo cual resulta sorprendente. La literatura científica disponible se centra principalmente en el estudio de la producción documental de la Unión Europea en su conjunto, como es el caso de la monografía elaborada por I. Thomson (1989), que ofrece la primera aproximación científica al tema o, en el caso español, la obra de Maciá (1996), que proporciona el estudio más completo realizado hasta el momento en nuestro país sobre el repertorio documental eurocomunitario. Otros trabajos publicados se centran en aspectos concretos de la documentación y la información europea, como es el caso del realizado por 
Grau Gaudix (1998a), donde se propone un análisis detallado de los documentos generados por la UE y una propuesta de clasificación de los mismos; el elaborado por Abiega (2000), que aporta información útil para identificar los distintos documentos producidos por las instituciones europeas así como sobre los datos que aparecen consignados en éstos, y el manual escrito por Martín González (2007), donde se detallan las distintas herramientas que facilitan la recuperación electrónica de la documentación y la información europea acompañada con casos prácticos.

En otros casos, los estudios se concentran en grupos documentales o herramientas informativas como pueden ser las publicaciones oficiales europeas (Masa y Salas, 1996), las bases de datos comunitarias (Fuentes García, 1993, 1994; Moreira, 2002), la producción documental de alguna institución comunitaria como es el caso de la obra escrita por Martín González (2002) sobre el Parlamento Europeo, así como el acceso electrónico a los documentos a través de Internet (Aleixandre Baeza, 1996; Grau Gaudix, 1998b; Viesca, 1998; Martín González, 2005).

En el conjunto de trabajos científicos publicados sobre los CDE se constata una ausencia significativa de investigaciones sobre sus recursos informativos. Así, en 1996, McCarthy publicó un breve estudio sobre el papel que juegan y las funciones que les son asignadas a los CDE. Ese mismo año Thomson (1996) presentó un alegato en favor de la presencia de este tipo de centros en los entornos universitarios. En 1997, Algarbani elaboró un trabajo sobre la red de centros de información y documentación europea en Andalucía en el que describe detalladamente la diferente naturaleza de estas unidades de información, entre ellas los CDE. También en esa fecha Marcella, Baxter, Parker y Davies profundizaron en el uso realizado por los usuarios de los centros de documentación europea de Francia y del Reino Unido. Finalmente, en 2000 surge un trabajo sobre los recursos que ofrecen estos centros (Olarán y Pérez-Trompeta, 2000) y sobre el acceso a ellos a través de medios electrónicos.

La elección del tema de investigación se justifica, por tanto, dada la notoria escasez de trabajos científicos realizados en el ámbito de la Bibliotecología sobre los recursos de información especializados que ofrecen los CDE y, en concreto, sobre su tipología y formas de organización.

Así, el propósito principal de nuestro trabajo es dibujar el mapa de recursos de información -entendidos como recursos fundamentales de cualquier organización o empresa (Moscoso, 1998) - que proporciona la Unión Europea a través de las páginas web de los Centros de Documentación Europea.

Con carácter más específico, este trabajo pretende igualmente: 
- Ofrecer un repertorio de fuentes de información digital que ayude a resolver la problemática que conlleva la localización de recursos documentales de la UE a las personas interesadas y/o estudiosas de la materia e hispanohablantes.

- Ordenar los distintos recursos especializados recopilados y proporcionar un modelo de clasificación de éstos.

- Dar a conocer a los interesados en la temática europea y/o pertenecientes a la comunidad hispanohablante los recursos informativos en formato electrónico elaborados por los centros de documentación europea en España.

\section{Metodología}

\section{Revisión bibliográfica}

El proceso de búsqueda de información se llevó a cabo utilizando distintas fuentes de información.

En primer lugar recurrimos a fuentes propias de la ciencia documental con el fin de localizar trabajos científicos publicados sobre el repertorio informativo y documental de la Unión Europea. Dado el carácter transnacional del ámbito objeto de estudio, consultamos principalmente obras monográficas así como revistas científicas con un amplio reconocimiento y difusión internacional. Asimismo se recurrió a bases de datos y repositorios del ámbito de la Biblioteconomía y la Documentación. Dada la patente escasez de trabajos realizados sobre el tema que nos ocupa procedimos, en segundo lugar, a la búsqueda de fuentes de información institucionales de la Unión Europea con el fin de aportarle una visión más completa a nuestra investigación. De este modo, consultamos el portal oficial de la Unión Europea, Europa; el sitio web de la red Europe Direct, la página web de la Oficina de Representación de la Comisión Europea en España y el catálogo ECLAS.

En todos los casos los términos de búsqueda elegidos fueron: "Centro de Documentación Europea”, "Unión Europea”, "recursos de información”, "mapas de recursos", "recursos electrónicos" e "información europea". Los términos se fueron combinando en ecuaciones utilizando operadores booleanos y operadores de proximidad.

Aunque nuestra idea inicial era acotar la búsqueda a la literatura científica más reciente, dada la ya comentada escasez de trabajos elaborados y/o publicados sobre la temática elegida, ampliamos la búsqueda al conjunto de estudios editados, con independencia de su fecha de edición. 


\section{Selección de la muestra}

El método elegido para realizar nuestro estudio fue el muestreo. El total de Centros de Documentación Europea establecidos en España constituyen la muestra de la investigación.

Para la localización de los CDE se utilizaron dos fuentes de información distintas con el fin de comprobar que los datos ofrecidos fueran coincidentes.

La primera fuente utilizada fue el portal oficial de la Unión Europea, Europa (http://europa.eu). A través de él accedimos a la página web de la red Europe Direct (http://europa.eu/europedirect), de la que forman parte los centros de documentación. La segunda fuente consultada fue el sitio web de la Oficina de Representación de la Comisión Europea en España (http:// ec.europa.eu/spain). En este caso, a través del enlace Recursos y puntos de información europea para los ciudadanos y las empresas, localizamos la red de CDE sitos en nuestro país, ordenados alfabéticamente por el nombre de las Comunidades Autónomas en las que se ubican.

Consultadas ambas fuentes se confirmó la existencia de un total de 37 centros de documentación europea en España. Seguidamente, procedimos a averiguar si cada uno de ellos contaba con página web y, por tanto, si permitía el acceso a sus recursos informativos en formato digital.

La localización de la dirección URL de los CDE fue tarea fácil porque esta información es proporcionada por la Oficina de la Comisión Europea en España a través del enlace citado en líneas anteriores. Se ofrecen además otros datos, tales como la dirección postal, el número de teléfono y de fax, el nombre del director del centro y del documentalista responsable, su dirección de correo electrónico y el horario de atención al público. En el caso del sitio web de la red Europe Direct solamente se facilita la dirección postal, el número de teléfono del centro y su correo electrónico.

El siguiente paso consistió en comprobar si se permitía el acceso digital a los centros, lo que fue posible en la mayor parte de los casos.

Los CDE de la Universidad de Las Palmas de Gran Canaria, de la Universidad Nacional de Educación a Distancia (UNED) y de la Universidad de Navarra no disponen de portal web. En el caso de la Universidad de La Coruña el servidor donde se encuentra alojada la página web del CDE no respondió en ninguna de las reiteradas veces en que intentamos acceder. Por otra parte, el acceso digital a los recursos informativos de los dos CDE con que cuenta la Universidad Complutense de Madrid se realiza a través de un mismo portal.

Estas circunstancias hicieron que se redujera a 32 el número de centros de documentación europea que consideramos. 


\begin{tabular}{|c|c|}
\hline Universidad - CDE & Enlace \\
\hline Universidad de Córdoba & http://www.uco.es/webuco/cde/index2.htm \\
\hline Universidad de Granada & http://cde.ugr.es/ \\
\hline Universidad de Sevilla & http://centro.us.es/cde/ \\
\hline Universidad de Zaragoza & http://www.unizar.es/derecho/cde/cde.html \\
\hline Universidad de Oviedo & http://www.uniovi.net/cde \\
\hline $\begin{array}{l}\text { Centre Balears Europa } \\
\text { (Palma de Mallorca) }\end{array}$ & http://www.documentaciocbe.eu/ \\
\hline Universidad de La Laguna & http://www.cdeuropea.ull.es/ \\
\hline $\begin{array}{l}\text { Universidad de } \\
\text { Cantabria (Santander) }\end{array}$ & http://www.buc.unican.es/CDE/ \\
\hline $\begin{array}{l}\text { Universidad Autónoma de } \\
\text { Barcelona }\end{array}$ & http://www.uab.cat/biblioteques/cde/ \\
\hline $\begin{array}{l}\text { Escuela Superior de } \\
\text { Administración y } \\
\text { Dirección de Empresas (ESADE) }\end{array}$ & http://www.esade.edu/cde/ \\
\hline Universidad de Gerona & http://biblioteca.udg.es/info_general/unitats/cde/ \\
\hline Universidad de Lleida & http://www.sbd.udl.cat/sbd/cde.html \\
\hline $\begin{array}{l}\text { Universitat Rovira i Virgili } \\
\text { (Tarragona) }\end{array}$ & http://www.urv.cat/biblioteca/cde/index.html \\
\hline $\begin{array}{l}\text { Universidad de Castilla } \\
\text { La Mancha (Toledo) }\end{array}$ & http://www.uclm.es/centro/ceuropeos/ \\
\hline Universidad de Salamanca & http://cde.usal.es/ \\
\hline Universidad de Valladolid & http://www.cdoce.uva.es/ \\
\hline CDE Extremadura (Badajoz) & http://www.cdiex.org/ \\
\hline $\begin{array}{l}\text { Universidad de Santiago de } \\
\text { Compostela }\end{array}$ & http://www.usc.es/es/servizos/cede/index.html \\
\hline $\begin{array}{l}\text { Universidad Complutense de } \\
\text { Madrid }\end{array}$ & http://www.ucm.es/BUCM/be/ \\
\hline $\begin{array}{l}\text { Universidad Politécnica de } \\
\text { Madrid }\end{array}$ & $\begin{array}{l}\text { http://www.upm.es/institucional/UPM/Biblioteca/NuestraBiblioteca/ } \\
\text { BibliotecasCentros/CEYDE }\end{array}$ \\
\hline Universidad San Pablo CEU & $\begin{array}{l}\text { http://www.uspceu.es/pages/servicios/cde/centro-documentacion-euro } \\
\text { pea-home.html }\end{array}$ \\
\hline Universidad Francisco de Vitoria & http://www.ufv.es/investigacion.aspx?sec=291 \\
\hline Universidad de Alcalá de Henares & http://www.uah.es/biblioteca/biblioteca/cde04.html \\
\hline Universidad Autónoma de Madrid & http://biblioteca.uam.es/cdeuropea/default.html \\
\hline Universidad Carlos III & \begin{tabular}{|l} 
http://www.uc3m.es/portal/page/portal/biblioteca/sobre_la_biblioteca/ \\
colecciones/nuestras_colecciones/centro_documentacion_europea
\end{tabular} \\
\hline Universidad de Murcia & http://www.um.es/biblio/cde/ \\
\hline $\begin{array}{l}\text { Universidad del País Vasco } \\
\text { (Bilbao) }\end{array}$ & $\begin{array}{l}\text { http://www.ehu.es/p200-content/es/contenidos/informacion/cde_centro_ } \\
\text { documetacin_europea/es_cde/principal.html }\end{array}$ \\
\hline Universidad de Deusto (Bilbao) & \begin{tabular}{|l} 
http://www.iee.deusto.es/servlet/Satellite/Page/1116406939495/_cast \\
/\%231116406939495/UniversidadDeusto/Page/facultadesTPL
\end{tabular} \\
\hline Universidad de La Rioja (Logroño) & http://biblioteca.unirioja.es/cde/index.shtml \\
\hline Universidad de Alicante & http://www.cde.ua.es/ \\
\hline Universidad Jaume I (Castellón) & http://sic.uji.es/cd/cde/ \\
\hline Universidad de Valencia & http://cde.uv.es/ \\
\hline
\end{tabular}




\section{Recolección y análisis de los datos}

Los datos para la realización de nuestro estudio se extrajeron mediante la consulta de cada uno de los portales web de los CDE. Durante este proceso comprobamos que algunas URL aportadas por la Oficina de Representación de la Comisión no eran correctas. Tal fue el caso del CDE de la Universidad Autónoma de Madrid, del CDE de la Universidad de Murcia y del CDE de la Universidad de Deusto. En otras ocasiones, la Comisión no ofrecía el sitio web del centro, lo que daba a entender que éste no existía. En esos casos, decidimos realizar nuestra propia búsqueda y comprobamos que tal dato era incorrecto, tal como ocurrió con el CDE de la Universidad de Cantabria que sí proporciona acceso electrónico a sus recursos.

Una vez localizadas y verificadas todas las direcciones web procedimos a extraer un listado de recursos informativos proporcionados por los centros con el fin de identificar la existencia de un patrón o modelo que se repitiera. Nuestra intención inicial fue considerar -casi de modo exclusivolos recursos agrupados bajo enlaces cuya denominación fuera la expresión "Fuentes de información en Internet", "Recursos de información" o similar. Sin embargo, la consulta detallada de los distintos apartados que conforman la estructura de las webs nos llevó a ampliar nuestro ámbito de estudio al comprobar que existían otros muchos recursos fuera de este grupo y que también constituyen fuentes de información que pueden tenerse en cuenta.

Por último procedimos a diseñar un modelo de agrupación que permitiera ordenar los recursos informativos, siguiendo una estructura sistematizada basada en grandes categorías.

Aunque fueron varios los criterios barajados para la elaboración del modelo, finalmente se optó por clasificar los recursos según el tipo de información que proporcionan, puesto que esta opción facilita considerablemente la tarea de recuperación de información llevada a cabo por el usuario. De este modo, se identificaron las categorías siguientes:

- Recursos de información jurídica

- Recursos de información institucional

- Recursos de información de actualidad

- Recursos de información educativa

- Recursos de información bibliográfica

- Recursos de información financiera

- Recursos de información estadística

- Recursos de información 2.0 


\section{RESULTADOS Y DISCUSIÓN}

El número así como la tipología de recursos informativos proporcionados por los centros de documentación europea resulta bastante significativo. Algunos centros han confeccionado incluso sus propias guías de recursos. Así, el CDE de la Universidad Autónoma de Barcelona ha elaborado un repertorio de recursos de información bastante completo en el que se incluyen no sólo fuentes de información oficial de la UE sino otro tipo de fuentes relacionadas con la temática europea. También la Universidad de Sevilla ha realizado una recopilación de fuentes de información europea que ofrece a modo de guía. No obstante, la elaboración de este tipo de herramientas no constituye una práctica habitual entre los CDE.

Existen otras situaciones como la del CDE de la Universidad Autónoma de Madrid, que ofrece en su web únicamente información sobre el centro pero no permite el acceso a ningún tipo de recurso ni de servicio en línea.

Es importante señalar que prácticamente el $90 \%$ de los recursos informativos que proporcionan los CDE son suministrados por el portal oficial de la Unión Europea, Europa, principal fuente de información sobre temática europea. De este modo, se reafirma el papel infomediario de los centros cuya labor consiste en redirigir al usuario hacia la fuente de información primaria. Por el contrario, se constata una significativa escasez de recursos de elaboración propia porque éstos se reducen, en su mayoría, a la elaboración de boletines informativos.

En las siguientes líneas, ofrecemos el mapa (Figura 1) de recursos informativos de los CDE españoles agrupados por categorías, establecidas éstas en función del tipo de información que ofrecen. En cada caso se recogen aquellos recursos que se proporcionan de forma repetida por todos los centros así como aquellos que destacan de un modo significativo y pueden ser, por tanto, objeto de especial interés para el usuario.

\section{Recursos de información jurídica}

La Unión Europea produce una gran cantidad de información jurídica, fruto, por un lado, de su capacidad legislativa, y por otro, de sus atribuciones jurisprudenciales.

Los recursos de carácter normativo a los que se puede acceder desde prácticamente todos los $\mathrm{CDE}$ son:

- El Diario Oficial de la UE (DOUE): es el boletín oficial de la Unión Europea. Se publica, en formato electrónico y a diario, en las lenguas 


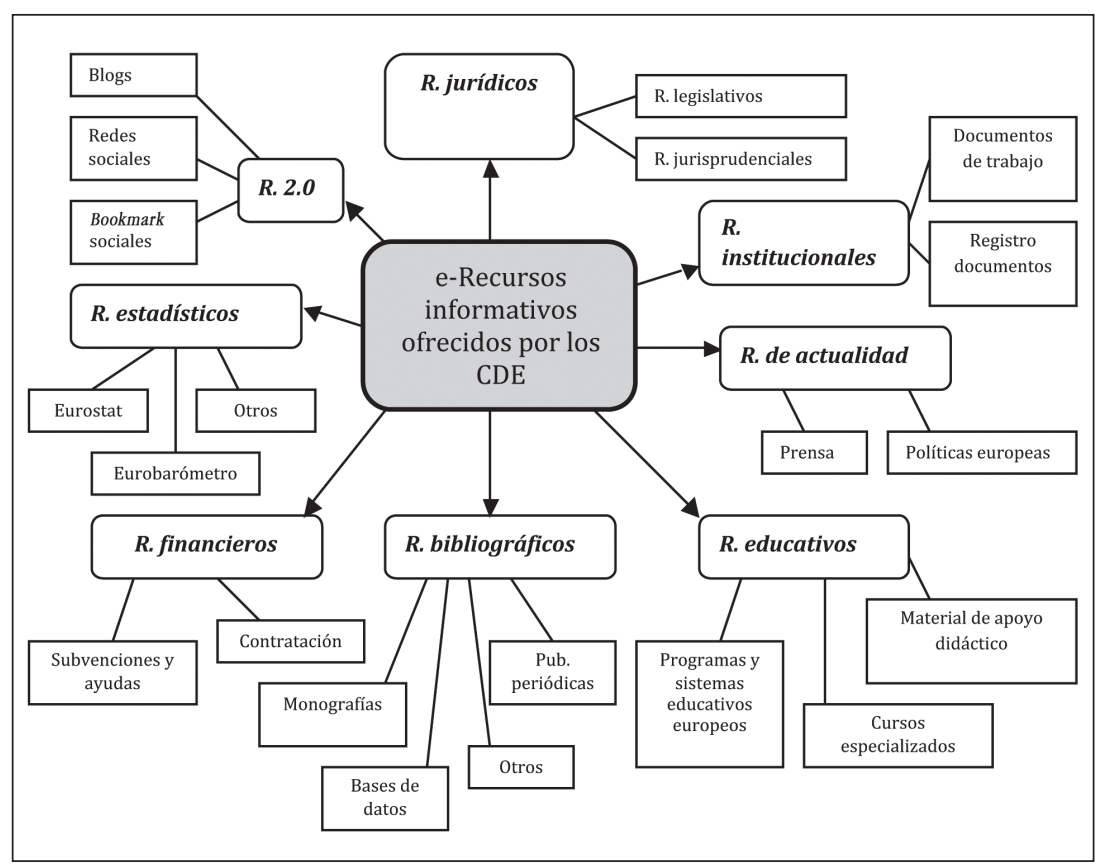

Figura 1. Mapa de recursos informativos ofrecidos por los Centros de Documentación Europea

oficiales de los Estados miembros. Se organiza en series. La Serie L contempla el conjunto de actos normativos aprobados por las instituciones con poder decisorio; es decir, reglamentos, directivas, decisiones, recomendaciones y dictámenes. La serie $\mathrm{C}$ aglutina las distintas comunicaciones e informaciones suministradas por las instituciones y órganos europeos. La serie $\mathrm{S}$ contiene anuncios de concursos públicos disponibles también en la base de datos TED.

- EUR-Lex: constituye el portal web principal del derecho de la Unión Europea. Da cabida a los trabajos preparatorios correspondientes a las distintas etapas del proceso legislativo y presupuestario así como a la documentación de carácter normativo originada en las instituciones. Esto es, las propuestas legislativas de la Comisión (documentos COM y SEC), dictámenes de los comités consultivos, posiciones comunes del consejo, reglamentos, decisiones, iniciativas parlamentarias, etc. Asimismo, permite la consulta de distintas colecciones documentales como tratados, acuerdos internacionales, legislación vigente y consolida$\mathrm{da}$, jurisprudencia y preguntas parlamentarias, entre otros.

- PreLex: es la base de datos que aglutina la documentación generada durante el seguimiento de los procedimientos interinstitucionales. De 
actualización diaria, esta herramienta ofrece todos los textos oficiales que van surgiendo durante el proceso legislador (propuestas, recomendaciones o comunicaciones) transmitidos por la Comisión a las instituciones con poder de decisión (Consejo y el Parlamento) así como a los órganos consultivos.

- Repertorio de la legislación comunitaria vigente: se trata de un índice de fuentes y referencias de la legislación comunitaria que contiene los actos comunitarios -básicamente el derecho derivado- en vigor. En él se pretende dejar patentes las modificaciones realizadas en los actos legislativos, de ahí que reúna las referencias a los textos iniciales y las modificaciones posteriores en el caso de haberlas.

- Sintesis de la legislación de la UE: se trata de una base de datos cuyo objetivo consiste en ofrecer una síntesis de la legislación comunitaria en todos y cada uno de los ámbitos de actuación comunitarios. Es gestionada por la Comisión Europea y se dirige a especialistas o a personas interesadas en las políticas y el funcionamiento de la Unión Europea. Este no es un recurso habitual en los sitios de los CDE consultados aunque sí lo ofrezca, por ejemplo, el centro de la Universidad de Lleida.

- Observatoire législatif (OEIL) del Parlamento Europeo: accesible únicamente en inglés y francés, reúne toda la información concerniente a los procedimientos normativos, presupuestarios y no legislativos en los que participa la Eurocámara así como resoluciones, iniciativas y procedimientos de organización interna de la institución.

En cuanto a la jurisprudencia emanada de los Tribunales eurocomunitarios, los recursos más habituales en los portales web de los CDE españoles son:

- CURIA: constituye la principal herramienta de consulta a la jurisprudencia eurocomunitaria pues permite el acceso electrónico al conjunto de sentencias, autos, conclusiones, informes y comunicaciones del Tribunal de Justicia, del Tribunal General y del Tribunal de la Función Pública europeos.

- Portal europeo e-Justicia es un servicio de ventanilla única a la jurisprudencia europea. A diferencia de lo que ocurre con el resto de recursos son pocos los CDE, como por ejemplo el de la Rioja, que ofrecen este portal entre sus recursos jurisprudenciales.

Algunos centros han elaborado su propia guía de recursos de derecho comunitario como la Universitat Rovira i Virgili. Sin embargo, hay otros como el 
de la Universidad de Córdoba que, al estar más orientado hacia la información económica, no ofrece ningún tipo de recurso jurídico a sus usuarios, lo que resulta bastante curioso. Otros incluso dan un paso más y ofrecen, como el CDE de la Universidad de Alicante, información organizada por temas sobre las transposiciones y aplicaciones de la normativa eurocomunitaria a la legislación española.

\section{Recursos de información institucional}

Los documentos elaborados por las instituciones y órganos comunitarios constituyen un recurso frecuente en los portales de los CDE debido al interés que despiertan para los estudiosos de los asuntos europeos. Conforman este grupo el conjunto de documentos generados por las instituciones en el cumplimiento de las competencias otorgadas por los distintos tratados comunitarios. Algunos centros incluso han elaborado listas organizadas de documentos institucionales con el fin de facilitar su acceso al usuario tal como ocurre en el caso del CDE de la Universidad de La Laguna. Otros recursos institucionales habituales son:

- Informe General sobre la actividad de la UE: es presentado cada mes de febrero a la Eurocámara con el fin de informar no sólo a la institución destinataria sino a la opinión pública sobre los acontecimientos pretéritos y los proyectos de futuro de las instituciones y órganos comunitarios y, especialmente, sobre las políticas comunitarias.

- Boletín de la UE: esta publicación recogía mensualmente y de forma resumida las actividades realizadas por las instituciones eurocomunitarias. El último boletín se editó en el mes de agosto de 2009; sin embargo, algunos centros permiten la consulta retrospectiva. A partir de esa fecha la información que ofrecía se publica a través del servicio Newsroom de la UE.

- Libros Verdes: son documentos de reflexión publicados por la Comisión sobre un ámbito político específico con los que se pretende lanzar una consulta a nivel europeo.

- Libros Blancos: son documentos que contienen propuestas de acción comunitaria en un ámbito específico.

Un recurso imprescindible en el acceso a la documentación institucional son los Registros de documentos. Se trata de una herramienta creada en 2001, en el marco normativo del derecho de acceso público a los documentos de la UE, que proporciona las referencias a los documentos elaborados por cada institución con el fin de que sean más fácilmente localizables para los ciudadanos. 


\section{Recursos de información de actualidad}

En esta categoría hemos incluido dos grupos de recursos distintos: en primer lugar, los recursos elaborados para los medios de comunicación y, en segundo lugar, la información relativa a las distintas políticas y actividades llevadas a cabo por la UE. En ambos casos se trata de información sobre noticias y temas de actualidad en la Unión Europea.

Por lo que respecta al primer grupo, las noticias, los comunicados de prensa y la agenda europea constituyen los principales pilares de la información de actualidad de la UE y son facilitados de un modo completo y actualizado por el CDE de la Universidad de Valladolid desde su página de acogida.

Los recursos más habituales en las webs de los CDE son:

- RAPID: base de datos del Servicio del Portavoz y una de las bases de datos históricas de la Comunidad. Da acceso a los comunicados de prensa emitidos por la UE con el fin de que los medios de comunicación informen sobre las distintas políticas y actividades llevadas a cabo por Bruselas.

- Newsroom: es la sala de prensa virtual oficial de la UE. Ofrece información de actualidad a los medios acreditados en Bruselas, así como material que pueda facilitar la labor de los periodistas, en general. Ciertos CDE como el de la Universidad de Lleida permiten además el acceso a las salas de prensa de la Comisión Europea y del Parlamento Europeo.

- Euronews: es un servicio de información periodístico que incluye noticias sobre Europa.

- Aqui Europa: diario digital, de origen español pero elaborado en Bruselas, que ofrece noticias sobre lo que acontece en Europa.

La Universidad de Salamanca ofrece un amplio y actualizado listado de recursos informativos para el personal de los medios de comunicación. Así, facilita la consulta a Euronews, Aqui Europa y Euroalert, entre otros. Sin embargo, destaca la base de datos, elaborada por la Universidad Complutense de Madrid, con noticias y artículos de opinión publicados sobre Europa en los principales periódicos españoles desde 1998 y en los diarios internacionales desde 2003.

Además de los recursos periodísticos propiamente dichos, los CDE proporcionan acceso a dossieres con información denominada "de actualidad", como son: información sobre la presidencia de turno de la UE, el Tratado de Lisboa o la celebración del Año Europeo. 
El otro grupo de información que conforma esta categoría es la denominada "temática" por la mayoría de los CDE y que hemos incluido para dar cuenta puntualmente de las diferentes actividades y políticas llevadas a cabo por la UE. El amplio repertorio de recursos electrónicos ofrecido por los CDE se organiza en 20 categorías temáticas siguiendo la clasificación de la Dirección General de Comunicación de la Comisión Europea. Puede verse un ejemplo consultando el CDE de Lleida y el de Salamanca.

\section{Recursos de información educativa}

Entre los recursos de carácter educativo puestos a disposición por los CDE se distinguen hasta tres grupos de información diferente.

En primer lugar se proporciona acceso a recursos sobre programas y sistemas educativos en Europa. En este caso los centros facilitan el acceso a servicios tales como:

- PLOTEUS, web oficial de la UE en materia educativa y de oportunidades de estudio en Europa.

- Información sobre programas europeos de intercambio: Erasmus, Tempus, Acción Jean Monnet

- Prácticas en las instituciones y órganos comunitarios. El CDE de la Universidad de Deusto proporciona acceso a las distintas páginas institucionales con información sobre la posibilidad de realizar prácticas remuneradas o no.

En segundo lugar se ofrece un elenco de cursos, másteres y actividades formativas que pueden estar organizadas por el propio centro o no. Sobresale en este sentido el CDE de la Universidad de Cantabria, que ofrece un catálogo exhaustivo de cursos formativos propios sobre temáticas variadas relacionadas con la UE.

En tercer lugar, algunos centros proporcionan recursos de apoyo a la docencia y a la investigación. Casi todos los que conforman este grupo minoritario permiten el acceso al sitio El Rincón del Profesor puesto en marcha por la propia Unión Europea. Otros, como el CDE de la Universidad de La Laguna facilitan información sobre el Espacio Europeo de Educación Superior (que también la ofertan el CDE de la Universidad de Cantabria y de la Universidad de Valladolid) y el Espacio Europeo de Investigación (la Universidad Complutense de Madrid facilita el acceso a la colección digital de la biblioteca central de la Comisión Europea así como información sobre comités y organizaciones de normalización, oficinas de patentes y convocatorias europeas para proyectos de investigación). 
Destaca como proveedor de recursos didácticos y centro de apoyo a la enseñanza el CDE de Tarragona, donde se proporciona acceso a las asignaturas sobre integración europea impartidas en la universidad. De cada materia, el centro facilita la guía didáctica, una bibliografía básica y un listado de recursos de información del CDE que puedan facilitar la tarea del profesor y del alumno en el estudio de la asignatura.

También resalta en este apartado el aula virtual que ofrece el CDE de la Universidad de Granada para dar apoyo electrónico a los cursos que organiza de forma presencial.

Por último, los CDE de Sevilla, Córdoba y Granada han creado una herramienta, euroexpertos.es, que ayuda a localizar a personal docente e investigador experto en temas europeos en las universidades andaluzas.

\section{Recursos de información bibliográfica}

Dentro del amplio número de recursos bibliográficos y documentales que suministran los $\mathrm{CDE}$ tenemos que distinguir los siguientes grupos: monografías, publicaciones periódicas, bases de datos y otros recursos.

Prácticamente todos los centros de documentación europea consultados les permiten a sus usuarios el acceso a las monografías editadas por la Oficina de Publicaciones Oficiales de la Unión Europea (EUR-OP) y a las suministradas por el sitio EUBookshop. Los centros también facilitan la consulta de publicaciones monográficas concebidas fuera del ámbito institucional europeo como hace el CDE de la Universidad Autónoma de Barcelona, que pone a disposición de sus usuarios las obras recibidas mediante donación de diferentes instituciones y las adquiridas a editoriales independientes entre las que se encuentra Cambridge University Press, Springer y Oxford University Press, entre otras.

En las publicaciones periódicas cabe distinguir dos subgrupos: las revistas científicas especializadas y los boletines informativos.

En la consulta a las publicaciones científicas destaca el acceso que proporciona la Universitat Rovira i Virgili a los sumarios de revistas especializadas en Derecho y Economía eurocomunitaria elaborados por la New York University. La Universidad de Castilla y La Mancha y la Universidad Politécnica de Madrid facilitan también la consulta temática de revistas electrónicas especializadas en asuntos europeos aunque el listado en el primer caso no es muy exhaustivo.

Por otra parte, los centros elaboran boletines electrónicos que pueden ser de varios tipos. Existen boletines de novedades (como el del CDE de la Universidad de Sevilla) y boletines informativos. En esta categoría destaca especialmente 
el boletín electrónico del CDE de la Universidad de Valencia al que cualquier usuario se puede suscribir y de esta forma permanecer semanalmente informado de las principales novedades así como de los temas de actualidad surgidos en el panorama europeo. El CDE de la Universidad de Alicante edita un boletín para sus usuarios más jóvenes: Menudas noticias de Europa. El CDE de la Universidad de Oviedo elabora además un repertorio de sumarios de revistas recibidas en el centro.

Destaca, por último, el catálogo de libros y revistas duplicadas que ofrece el CDE de la Universitat Rovira i Virgili a disposición de sus usuarios.

Las bases de datos constituyen un recurso informativo recurrente en el mapa de recursos de los CDE. Por lo general éstos facilitan la consulta de las bases de datos puestas a disposición por la Unión Europea y organizadas de dos formas distintas, bien por temas bien por orden alfabético. En el caso del CDE de Lleida únicamente permite el acceso directo a la base de datos terminológicos IATE, mientras que el CDE de la Universidad de Valladolid cuenta con sus propias bases de datos, una de títulos y sumarios de revistas y otra de monografías (últimas adquisiciones), todas ellas especializadas en la UE.

Otros recursos a los que los CDE permiten el acceso con mayor o menor frecuencia son:

- Who's who, el directorio de la UE (puede accederse desde el CDE de La Rioja).

- ECLAS, el catálogo bibliográfico de la Comisión Europea (puede consultarse desde la Universidad de La Laguna).

- EUTube, sitio con documentación multimedia de temática europea. La universidad riojana proporciona documentación audiovisual especializada en Europa a través de la Filmoteca Europea.

- Repositorios documentales con recursos especializados en temas comunitarios. El CDE de la Universidad de Alcalá facilita acceso a E-SciDR, ESO (European Sources Online) o Archidok. El centro de la Universidad de Santiago de Compostela permite la consulta al proyecto internacional European Research Papers Archive y a otros repositorios de origen no europeo, como son el AEIplus de la Universidad norteamericana de Pittsburg.

- Folletos, mapas, carteles y todo tipo de información divulgativa es suministrada por EUR-OP a través de los CDE en formato electrónico y también impreso. 


\section{Recursos de información financiera}

Incluimos en esta categoría el conjunto de recursos informativos sobre subvenciones, ayudas y préstamos así como sobre programas y acciones de financiación europea. Los más frecuentes son:

- EPSO: Oficina Europea de Selección de Personal, facilita información sobre vacantes de empleo en las instituciones, órganos y agencias comunitarias.

- EURES: es el sitio sobre las oportunidades de empleo de la UE puesto a disposición de las empresas y de los ciudadanos, y al que permiten su consulta la Universidad de Deusto, entre otras.

- TED: base de datos con información sobre contratación pública.

Algunos centros ofrecen otros recursos como son:

- SIMAP: portal europeo de contratación pública al que proporciona acceso el CDE de la Universidad Complutense de Madrid.

- CORDIS: Servicio de Información Comunitario sobre Investigación y Desarrollo.

- INFOREGIO: servicio sobre política de inversiones en el ámbito regional (Fondos FEDER, FSE y Fondo de Cohesión).

- Guía de financiación comunitaria: resulta más que recomendable el catálogo de recursos sobre ayudas comunitarias, instrumentos financieros y búsqueda de socios para la realización de proyectos de investigación, ofrecido por la Universidad de Valencia. La Universidad de Cantabria también facilita su consulta.

- Guía de ayudas de la UE: elaborada por la Diputación de Barcelona para las corporaciones locales y ofrecida por el CDE de Valencia.

Dada la orientación económica que ostenta el Centro de Documentación e Información de Extremadura, ofrece una interesante información sobre convocatorias de financiación europeas -se facilita la consulta a través de un buscador-, una mensajería de programas que permiten estar puntualmente informados de los programas europeos que disponen de plazos muy cortos para la presentación de candidaturas. Como nota curiosa quisiéramos apuntar la información actualizada que ofrece este centro sobre el tipo de cambio del euro. 


\section{Recursos de información estadística}

Aunque este tipo de información es ofrecida prácticamente por la totalidad de los centros de documentación consultados, los recursos estadísticos son más bien escasos y responden básicamente a dos fuentes:

- Eurostat: agencia encargada de elaborar las estadísticas oficiales, generales y especializadas, de la UE. El CDE de la Universidad de Extremadura muestra las distintas colecciones de estadísticas que elabora Eurostat y que a su vez se presentan en series agrupadas en torno a distintos temas.

- Eurobarómetro: publicación que recoge las distintas encuestas de opinión realizadas sobre asuntos europeos.

- Cifras clave sobre Europa: consiste en una serie de estadísticas básicas relativas a la UE.

Algunos centros añaden además la información estadística elaborada por el Banco Central Europeo quizás porque consideran que puede ser de interés en el momento actual de crisis económica.

Otros recursos estadísticos proceden del Instituto Nacional de Estadística, del Instituto de Estadística de la Comunidad de Madrid (se pueden consultar desde el CDE de la Universidad Politécnica de Madrid), del barómetro del Real Instituto Elcano y del Centro de Investigaciones Sociológicas (la consulta de estos últimos y de nomenclaturas y glosarios de términos estadísticos es facilitada por la Universidad Carlos III).

El CDE de la Universidad de Valladolid redirige a sus usuarios hacia el buscador y a los directorios de datos estadísticos que elabora la Universitat Pompeu Fabra.

\section{Recursos de información 2.0}

Al amplio listado de recursos que ofertan los Centros de Documentación Europea se ha incorporado la denominada información 2.0, información que el usuario maneja en tiempo real y que además puede alimentar con sus propias aportaciones. Por otra parte, estas unidades de información se sirven de las tecnologías 2.0 para conseguir una mayor difusión de los recursos, servicios y actividades que realizan. Así, encontramos:

- Blogs: la Universidad Autónoma de Madrid propone un blog de convocatorias del centro de documentación donde, a modo de tablón de 
anuncios, ofrece información sobre becas, ayudas, seminarios, congresos, etc. Acceso a blogs sobre temas europeos lo ofrecen, de momento, la Universidad de La Laguna (que distingue entre blogs institucionales y blogs especializados) y la Universidad de Valencia.

- Bookmarks sociales: la Universidad Autónoma de Barcelona utiliza la herramienta Delicious para agrupar los sitios web favoritos con recursos temáticos sobre Europa.

- En las redes sociales ya están presentes el Centro de Documentación Europea extremeño, que mantiene una cuenta en Facebook, y el Centro de la Universidad Francisco Vitoria y el de la Universidad de Valencia que hacen lo propio en Twitter.

- Sindicación a sus contenidos (RSS) permiten el Centro de Documentación de Extremadura, el de la Universidad de Santiago de Compostela y el de la Universidad de Valencia (este centro ofrece también el acceso a su información mediante etiquetas).

\section{Conclusiones}

En los últimos años la UE ha mostrado una constante preocupación por proporcionar acceso a su información. Dicha tarea se ve facilitada por las distintas unidades de información entre las que se encuentran los Centros de Documentación Europea, que actúan en el ámbito universitario como intermediarios entre las fuentes de información especializadas y los ciudadanos.

Con el fin de mejorar su labor de difusión informativa, los Centros de Documentación Europea han creado sus propios sitios web desde los que ofrecen, en soporte electrónico, acceso a sus fuentes y a sus servicios documentales. Dichas páginas, fácilmente accesibles y bien estructuradas en general, permiten la consulta de un amplio conjunto de recursos que se repite en prácticamente todos los centros a modo de patrón. No obstante, existen diferencias entre las webs de unos centros y otros ya que podemos encontrarnos desde portales cuya información está desactualizada (CDE de Gerona) o es deficiente (CDE de Zaragoza) hasta portales muy ricos en recursos (CDE de Salamanca) e incluso que incorporan tecnologías 2.0 (CDE de Valencia).

Por otra parte, al no existir ninguna disposición por parte de la Comisión Europea que obligue a los centros a ofrecer determinadas fuentes de información en sus páginas web, cada sede hace su propia selección de los recursos informativos que quiere ofrecer, aunque bien es cierto que en dicha selección se acusa cierta influencia del ámbito científico al que pertenezca el director/a del centro o de la facultad. 
Por lo que respecta al origen de los recursos se observa que, en su mayoría, son elaborados por la propia Unión Europea por lo que el CDE actúa como mero mediador entre la fuente original y el usuario. Esta circunstancia, repetida en los $\mathrm{CDE}$ de todo el mundo, se constata principalmente en el caso de los recursos de tipo jurídico, institucional, estadístico y de financiación donde los CDE recurren a las páginas web oficiales de las instituciones y agencias europeas cuando se trata de ofrecer información. El resto lo conformarían aquellos recursos que son elaborados por el propio centro o que no proceden de ningún organismo comunitario, lo que suele ser frecuente en el caso de los recursos bibliográficos, educativos y de los que hemos denominado 2.0. En este segundo grupo sobresalen los boletines informativos y los de novedades, al igual que los blogs especializados en temática europea.

En cuanto al grupo más numeroso de recursos ofertados, destaca el conjunto de recursos de información jurídica seguido del grupo de recursos de información institucional. Esta circunstancia resulta lógica si tenemos en cuenta la gran capacidad legislativa y jurisprudencial otorgada por los Tratados a la Unión Europea y su influencia en el ordenamiento jurídico de los Estados miembros. Por otra parte, el conjunto de documentos de trabajo de las instituciones europeas así como el acceso a los mismos mediante los registros documentales son demandados a menudo por los usuarios de los CDE. Son frecuentes también los recursos de información estadística generados por la Oficina de Estadísticas Europea (Eurostat) y los sondeos de opinión (Eurobarómetro) así como la información sobre financiación comunitaria y recursos de actualidad (desde sus orígenes, la Unión Europea ha demostrado una constante predisposición para informar a los medios periodísticos sobre sus políticas y actividades). La presencia de recursos bibliográficos y documentales es más que comprensible en el contexto universitario donde se enclavan los CDE y justificado además por su condición de impulsores de estudios académicos sobre Europa. Por el contrario, llama la atención que, pese a su ubicación en universidades donde se imparten materias y títulos especializados en la UE, sean escasos los recursos ofrecidos como apoyo a las tareas docente e investigadora.

Mención especial merece la escasa presencia de los CDE en redes sociales como Facebook y Twitter. Del total de centros estudiados, un porcentaje todavía muy bajo cuenta con presencia en la web 2.0 (CDE de la Universidad Francisco de Vitoria, el CDE de Valencia y el CDE de Extremadura) lo cual nos resulta a todas luces sorprendente pues ésta constituye, hoy por hoy, una importante herramienta de difusión informativa, especialmente entre la gente joven cuya presencia es mayoritaria en el ámbito universitario. Sin duda, la presencia de estas unidades de información en las redes sociales contribuirá 
en primer lugar al conocimiento de este tipo de centros y en segundo lugar, a través de la comunicación directa con sus internautas, a reconocer las necesidades de información de sus usuarios.

En definitiva, podemos concluir que los Centros de Documentación Europea conforman un importante punto de acceso a la información de la Unión Europea dado el amplio número de recursos que permiten consultar y su diferente naturaleza. No obstante, quizás su papel como transmisores de información deba ser replanteado puesto que se constata una fuerte tendencia a ofrecer fuentes de origen oficial siendo escasos los recursos especializados elaborados por el propio CDE y que, sin duda, serían los que aportasen a cada centro un valor añadido.

\section{Bibliografía}

Abiega Picatoste, M. (2000), La documentación de la Unión Europea, Madrid: Colex.

Aleixandre Baeza, E. (1996), “La Unión Europea en Internet”, en Métodos de información, vol. 3 (11-12), 39-46.

Algarbani Rodríguez, J. M. (1997), "La red de centros de información y documentación europea. Una visión desde Andalucía”, en Sistemas y politicas de información de las Autonomías. Situación actual y perspectivas, I Jornadas Andaluzas de Documentación, Sevilla: Asociación Andaluza de Documentalistas.

Fuentes García, J. (1993), "Política de información y bases de datos de la Comunidad Europea”, en Revista de las Cortes Generales, vol. 30, 161-178.

Fuentes García, J. (1994), "Análisis de los elementos estructurales de la Unión Europea: fuentes documentales y recuperación bibliográfica”, en Revista de las Cortes Generales, vol. 32, 243-270.

Grau Gaudix, R. M. (1998a), "Análisis de la documentación de la Unión Europea”, en Revista de las Cortes Generales, vol. 43 (1), 308-320.

_ (1998b), "El acceso electrónico de la documentación comunitaria", en Revista de las Cortes Generales, vol. 43 (1), 277-404.

Llorente Pinto, Ma. P. (2007), "Las redes de información eurocomunitarias: El Centro de Documentación Europea de la Universidad de Salamanca", en Y. Martín González (ed.), La información de la Unión Europea: política, sistema y redes, Universidad de Salamanca, Salamanca.

Marcella, R.; Baxter, G. y Parker, S. (1997), "The provision of European Information by public libraries in the UK", en Library Management, vol. 18 (9), 7-41.

Maciá, M. (1996), Documentación de la Unión Europea, Madrid: Síntesis. 
Marcella, R. et al. (1997), "The pattern of provision of European Union information in France and the United Kingdom: a comparative study of services", en Library Management, 18 (5), 243-252.

Martín González, Y. (2002), La documentación y los servicios de información del Parlamento Europeo, Gijón: Trea.

_ (2005), "Principales mecanismos para la localización y recuperación de la información de la Unión Europea”, en Cuadernos $B A D$, vol. 2, 64-73.

- (2007), Manual de documentación europea. Análisis y recuperación de la información eurocomunitaria, Gijón: Trea.

Masa Godoy, L. y Salas Olgado, J. C. (1996), "Las publicaciones oficiales de la Unión Europea”, en Métodos de información, vol. 3 (1112), 27-31

McCarthy, E. (1996), "The role of the European Documentation Centres", en Aslib Proceedings, vol. 43 (6), 203-205.

Moreira, A. (2002), "Las bases de datos de la Unión Europea”, en Revista Valenciana de Economía y Hacienda, vol. 5, 249-262.

Moscoso, P. (1998), "Reflexiones en torno al concepto 'Recurso de información' ”, en Revista General de Información y Documentación, vol. 8 (1), 327-342.

Olarán, M. y Pérez-Trompeta, Á. (2000), “La difusión documental en los Centros de Documentación Europea de España: el acceso a través de Internet, 2001. Actas", en VI Congreso de Cultura Europea, Pamplona, España: Universidad de Navarra, Centro de Estudios Europeos, 899-912

Sánchez Domínguez, Ma. C. (2001), "Modelos para la organización de las fuentes de información de la Unión Europea. Actas", en $V$ Congreso ISKO-España, Alcalá de Henares, España: Ana Extremeño Placer, 137-147.

Terra, A. L. (2009), "El multiculturalismo en la UE: organización y acceso a la información: el ejemplo de los centros de documentación europea. Actas", en IX Congreso ISKO-España, Valencia, España: Universidad Politécnica de Valencia, pp. 167-188.

_ (2010), "European information user. Topics from a comparative analysis within the European Union", en The new library world, vol. 111 (5/6), 189-202.

Thomson, I. (1989), The documentation of the European Communities. A guide, London: Mansell.

_ (1996), "Desafíos de los Centros de Documentación Europea", en Métodos de información, vol. 3 (11-12), 18-23.

Viesca, J. (1998), La Unión Europea en Internet, Madrid: Anaya multimedia. 